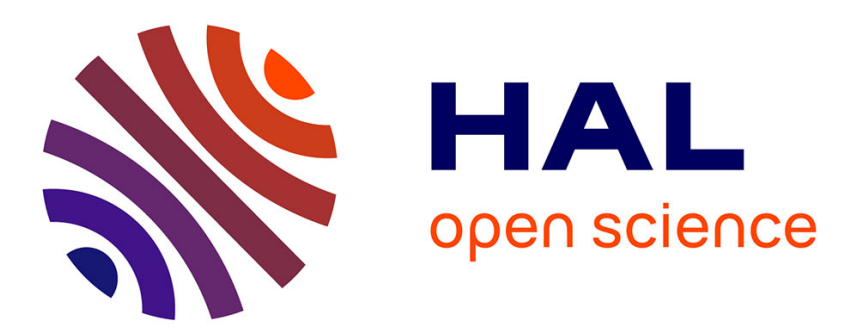

\title{
Torque Control of Electrorheological Fluidic Actuators
}

Marie-Aude Vitrani, Jason Nikitczuk, Guillaume Morel, Constantinos

Mavroidis

\section{To cite this version:}

Marie-Aude Vitrani, Jason Nikitczuk, Guillaume Morel, Constantinos Mavroidis. Torque Control of Electrorheological Fluidic Actuators. American Control Conference, Jun 2004, Boston, United States. pp.5072-5077. hal-01171247

\section{HAL Id: hal-01171247 \\ https://hal.science/hal-01171247}

Submitted on 9 Jul 2015

HAL is a multi-disciplinary open access archive for the deposit and dissemination of scientific research documents, whether they are published or not. The documents may come from teaching and research institutions in France or abroad, or from public or private research centers.
L'archive ouverte pluridisciplinaire HAL, est destinée au dépôt et à la diffusion de documents scientifiques de niveau recherche, publiés ou non, émanant des établissements d'enseignement et de recherche français ou étrangers, des laboratoires publics ou privés. 


\section{Torque Control of Electrorheological Fluidic Actuators}

\author{
${ }^{\text {A }}$ Laboratoire de Robotique de Paris (LRP) \\ 18, route du Panorama - BP 61 \\ 92265 Fontenay-aux-Roses Cedex \\ Tel. : (+33) 01465478 12; Fax : (+33) 0146547299 \\ Email: morel@robot.jussieu.fr, \\ Webpage: http://www.robot.jussieu.fr/ \\ ${ }^{1}$ Author for Correspondence

\begin{abstract}
In this paper, the experimental closed loop torque control of Electro-Rheological Fluids (ERF) based actuators for haptic applications is performed. ERFs are liquids that respond mechanically to electric fields by changing their properties, such as viscosity and shear stress, electroactively. Using the electrically controlled rheological properties of ERFs, we developed actuators for haptic devices that can resist human operator forces in a controlled and tunable fashion. In this study, the ERF actuator analytical model is derived and experimentally verified and accurate closed loop torque control is experimentally achieved using a non-linear proportional integral controller with a feed-forward loop.
\end{abstract}

Vitrani M. A. ${ }^{\mathrm{A}}$, Nikitczuk J. ${ }^{\mathrm{B}}$, Morel G. ${ }^{\mathrm{A}}$, Mavroidis C. ${ }^{\mathrm{B}, 1}$

\section{INTRODUCTION}

During the last fifty years, it has been known that there are liquids that respond mechanically to electrical stimulation. These liquids change their viscosity electroactively and they have attracted a great deal of interest of engineers and scientists. These ElectroRheological Fluid (ERF) exhibit a rapid, reversible and tunable transition from a fluid state to a solid-like state upon the application of an extemal electric field [1]. Some of the advantages of ERFs are their high yield stress, low current density, and fast response (less than 1 millisecond). ERFs can apply very high electrically controlled resistive forces while their size (weight and geometric parameters) can be very small. ERFs can be combined with other actuator types such as electromagnetic, pneumatic or electrochemical actuators so that novel, hybrid actuators are produced with high power density and low energy requirements [2]. The electrically controlled rheological properties of ERFs can be beneficial to a wide range of technologies requiring damping or resistive force generation. Examples of such applications are active vibration suppression and motion control. Several commercial applications have been explored, mostly in the automotive industry for ERF-based engine mounts, shock absorbers, clutches and seat dampers. Other applications
${ }^{B}$ Department of Mechanical and Industrial Engineering 375 Snell Engineering Center, Northeastern University 360 Huntington Avenue, Boston MA 02115 Tel: 617-373-4121, Fax: 617-373-2921 Email: mavro@coe.neu.edu Webpage: http://www.coe.neu.edu/ mavro

include variable-resistance exercise equipment, earthquakeresistant tall structures and positioning devices [1].

While a lot of work has been performed on the design, modeling and testing of ERF based actuators and devices; very little work has been performed on the closed loop control of these devices. All of the existing work in this area concerns the position control [3] while no work has been performed on the force / torque control of these devices. ERF devices are generally used with position control as dampers to avoid vibrations [4], such as in vehicle suspensions [5] or in anti-seismic buildings [6]. Position control has also been studied for ERF based valves [7].

Our group has developed and studied several ERF based actuators and haptic systems [8]. Recently we developed prototypes of rotary ERF based actuating elements for haptic knobs for vehicular instrument controls [9]. In this study, the ERF actuator analytical model is derived and experimentally verified and accurate closed loop torque control is experimentally achieved using a non-linear proportional integral controller with a feed-forward loop.

\section{FUNDAMENTALS OF ERF MODELLING}

ERF are suspensions of polarizable particles in viscous non-conducting oil with particle-fluid dielectric mismatch. Typical suspended materials in an ERF are $1-100 \mu \mathrm{m}$, approximately spherical, while the suspending fluids are non-conducting solvent [10]. When an uncharged particle is placed in an electric field, it develops an induced dipole if the surrounding medium has a different dielectric constant due to the differing polarizability of the two materials. This dipole is further enhanced by the presence of other particles since they intensify the local electric field experienced by any one particle. Particle polarization changes their organization in the fluid and causes changes in fluid rheological properties. The changes in the suspension microstructure increase the effective viscosity of a sheared ERF. This effect is called the Winslow effect. The effective 
viscosity of the ER suspension can be 100,000 times greater for electric field strengths of about $1 \mathrm{kV} / \mathrm{mm}$ perpendicular to the direction of flow.

Modelling the behaviour of an ERF is generally performed by using a known conventional fluid (or even solid-plastic) model, thus defining the basic structure of the model, and then characterizing how the model parameters are affected by the application of an electric field, or by the variation of some internal state variables, such as temperature or the type of flow [11]. Under zero field conditions ERFs are generally characterized by a simple Newtonian viscosity. When subjected to high electric fields, ERFs develop a yield stress and their shear stress $\tau$ is fairly well modelled as a Bingham plastic:

$$
\tau=\mu \dot{\gamma}+\tau_{y, d}
$$

where $\mu$ is the plastic viscosity, $\dot{\gamma}$ is the shear rate and $\tau_{y, d}$ is the Bingham or dynamic yield stress.

One can conclude by extrapolation to zero shear rate that the stress must exceed this dynamic yield stress in order for the material to flow, similarly to a dry coulomb friction model. In fact, the minimum stress required to cause the ERF to flow is not necessarily the dynamic yield stress but rather the static yield stress $\tau_{y, s}>\tau_{y, d}$. Using the friction analogy, this can be understood as the well-known Stribeck effect. More precisely, there are three yield stresses characterizing the behaviour of an ERF [12]. The first is the elastic-limit yield stress $\tau_{e}$, which is used, in solid mechanics. Upon complete removal of stress exceeding $\tau_{\mathrm{e}}$, the material never fully recovers and suffers a permanent strain $\gamma_{e}$, which is the transition between elastic and plastic deformation. The elastic-limit yield stress is not the limit of linear behaviour but rather the limit of reversibility for the material. Loss of linear behaviour generally occurs before the elastic limit. The static yield stress $\tau_{\mathrm{s}}$ is the minimum stress necessary for the unbounded strain or the deformation of the material. Finally the plateau stress for large strains is the dynamic yield stress $\tau_{d}$.

The Bingham plastic model has been widely used to predict the post-yield behaviour of ERF, i.e. the behaviour of the ERF when flowing. However, if the fluid experiences shear thinning or thickening, the Bingham plastic model may not be an accurate predictor of behaviour since the post-yield plastic viscosity is assumed to be constant. When shear thinning or thickening effect is more pronounced, the post-yield behaviour becomes non linear. In order to accurately model this non linear post-yield (or flowing) behaviour, another generic fluid model should be used that is called the Herschel-Bulkley model [13]:

$$
\tau=\tau_{y, \mathrm{~d}}+\mathrm{k} \dot{\gamma}^{n}
$$

This fluid model is a generalized model for viscoplastic flow with yield stress. It can be reduced to the Bingham plastic model (namely, $n=1$ ) in the case where post-yield shear thinning or thickening are minimal. Finally, the literature also provides global structural models aimed at describing both the non-flowing and the flowing behaviour of ERFs. They are generally formalized as a serial and parallel combination of springs and dampers, associated with some non-linear friction terms, and, eventually, some hysteretic elements.

In regard to the Bingham model described by Equation 1 , the applied electric field $E$ affects the dynamic yield stress, with a quadratic relationship. This yields:

$$
\tau_{y, d}=\alpha E^{2}
$$

The ERF used in this project is the LID 3354S manufactured by Smart Technology Ltd. [14]. It is made up of $35 \%$ by volume of polymer particles in silicone/fluorolube base oil. According to the provider, the field dependencies for this particular fluid are:

$$
\left\{\begin{array}{c}
\tau_{y, s}=C_{s}\left(E-E_{r e f}\right) \\
\tau_{y, d}=C_{d} E^{2} \\
\mu=\mu_{0}-C_{v} E^{2}
\end{array}\right.
$$

Where $\mu_{0}$ is the zero field viscosity, $C_{s} ; C_{d}, C_{v}$ and $E_{\text {ref }}$ are constants which approximate values are supplied by the manufacturer. Obviously, the formula for the static yield stress is only valid for fields greater than $\mathrm{E}_{\text {ref }}$. In this work, it is assumed that the torque controller will be used to finely control the resistive torque of a device that is moved by an operator (or by an auxiliary motor) and therefore only the dynamic mode is considered.

\section{EXPERIMENTAL SYSTEM}

A haptic feedback joystick and knob has been developed by our team to address the desire for simplifying and enhancing the human-vehicle interface [9]. These devices rely on passive, revolute ERF-based actuators to induce controllable resistive torques. These actuators are used in this paper as the test-bed to perform closed loop torque control experiments.

The studied ERF actuator consists of multiple parallel rotating electrode plates to enhance the resistive torque output capability of the actuator by increasing the activated area of the fluid while maintaining a compact volume. Figure 1 shows a cut-away view and a picture of the assembled multiple Flat-Plate or FP actuator. Two shafts are designed and situated in a concentric orientation with one shaft fixed to the housing of the actuator and the second able to rotate against the fixed shaft. Two different circular copper plates, designed to match the attachment points on each of the two shafts, are connected to the shafts and serve as the positive and negative electrodes that generate the electric field to actuate the ERF that sits inside 
the gap. The assembled shafts are then inserted into a housing that is filled with ERF and sealed to complete the assembly of the actuator. Considerable effort was made to reduce the friction caused by the seal. Applying an electric field across the gaps causes the fluid properties to change, resulting in an increase in yield stress. This property is used to control the force feedback of the actuator. The plastic parts of the prototype were built using rapid prototyping methods to allow for the quick creation of complicated shapes that would not have been possible with machine shop methods. The copper plates were machined, however, using more traditional methods. Table 1 shows the important parameters of the FP actuator prototype.
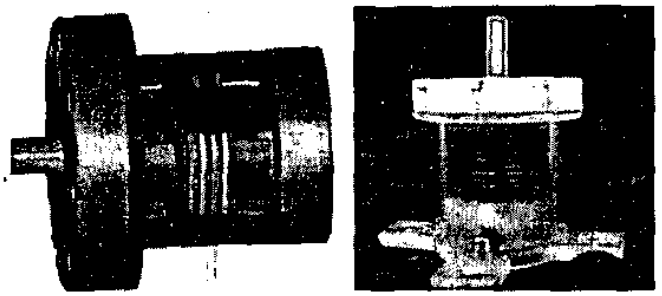

Fig. 1: Flat-Plate ERF Actuator: CAD Drawing \& Prototype.

To test the performance of the FP actuator prototype, to experimentally validate its theoretical model and to perform closed loop torque control experiments the actuator was incorporated into an experimental setup, shown in Figure 2. The actuator shaft is attached to a flexible coupling to ensure proper alignment. The other side of the flexible coupling is connected to a DC motor through a belt with a pulley wheel. This motor applies known external torques to the FP actuator that will controllably resist these externally applied torques. An optical encoder attached to the shaft is used to measure the angular displacement. A torque sensor is attached at the base of the FP actuator to measure its resistive torque output. In this experimental setup, two parameters can be changed by the user: the motor velocity and the voltage applied to the fluid.

TABLE 1: Summary of ERF Actuator Properties

\begin{tabular}{|l|l|}
\hline & FP actuator \\
\hline Outer radius & $14.7 \mathrm{~mm}$ \\
\hline Length & $32.5 \mathrm{~mm}$ \\
\hline Gap width & $.5 \mathrm{~mm}$ \\
\hline Dynamic Torgue at 2 kV & $188.5 \mathrm{mN} \mathrm{m}$ \\
\hline Dynamic Torque at 4 kV & $753.4 \mathrm{mN} \mathrm{m}$ \\
\hline Range of motion & $120^{\circ}$ \\
\hline Number of Plates & 15 \\
\hline
\end{tabular}

A PC augmented with a US Digital $P C 7166^{\mathrm{TM}} \mathrm{PC}$ to incremental encoder interface card and a Datelß PC$412 \mathrm{C}^{\mathrm{TM}}$ Analog I/O board was used in the open and closed loop experiments. The PC collects the sensor measurements, through the data acquisition board or the encoder interface card, performs the feedback control calculation and then it sends out the signal to the FP actuator, through the D/A converter and laboratory built amplifiers.

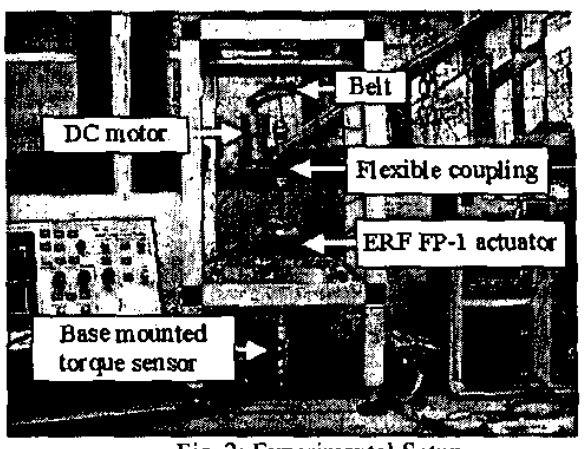

Fig. 2: Experimental Setup

\section{FP ACTUATOR MODEL}

Modelling of ERF actuators for use in closed loop torque control is inexistent. Therefore our first step was to develop a model of the ERF actuator used that is suitable for closed loop torque control. Figure 3 defines important geometric parameters that are used in the model. Since the system is mostly to be used when flowing, the post-yield model (i.e. dynamic mode) is derived, Providing an actuator model from the fluid model supposes to map the shear stress into the output torque and the shear rate into the output velocity. To do so, we assume that the shear stress is constant along a line parallel to the axis, between the electrodes. Also, due to the symmetry of the system, the shear stress is supposed to depend only on the radius $r$, not on the angle $\theta$.
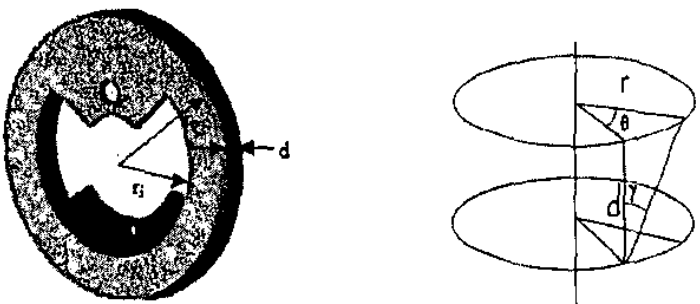

Fíg. 3: FP Actuator Model [mportant Parameters

The elementary resistive torque between a pair of electrodes is then:

$$
\mathrm{T}_{\text {ele }}=\int_{i}^{t_{0}} \mathrm{rr}(\mathrm{r}) \mathrm{dA}
$$

where $d A=2 \pi r d r$ and $\tau(r)$ is the shear stress at a radius $r$ from the axis.

Since $\mathrm{N}$ rotating plates are mounted in between of $\mathrm{N}+1$ fixed plates, there are $2 \mathrm{~N}$ gaps filled with $\mathrm{ERF}$, producing a total resistive torque given by:

$$
\mathrm{T}=2 \mathrm{NT}_{\text {ele }}=4 \pi \mathrm{N} \int_{\mathrm{i}}^{\mathrm{r}_{0}} \mathrm{r}^{2} \tau(\mathrm{r}) \mathrm{dr}
$$

Furthermore, for a displacement $\delta \theta$ of the actuator, the (angular) shear deformation of a fluid element $\delta \gamma$ (see Figure 3 ) at a radius $r$ is: 


$$
\delta \gamma=\frac{r}{d} \delta \theta
$$

The relationship from the actuator angular velocity to the shear rate is then:

$$
\dot{\gamma}=\frac{\mathrm{r}}{\mathrm{d}} \dot{\theta}
$$

Thus, combining Equations (1), (4), and (8), one gets:

$$
\tau(r)=C_{d} E^{2}+\left(\mu_{0}-C_{v} E^{2}\right) \frac{r}{d} \dot{\theta}
$$

Combining with Equation (6), one gets:

$$
T=4 \pi N \int_{i}^{T_{0}} r^{2}\left(C_{d} E^{2}+\left(\mu_{0}-C_{v} E^{2}\right) \frac{r}{d} \dot{\theta}\right) d r
$$

which finally leads to:

$$
T=4 \pi N\left[C_{d} \frac{r_{0}^{3}-r_{i}^{3}}{3}-C_{v} \frac{r_{0}^{4}-r_{i}^{4}}{4 d} \dot{\theta}\right] E^{2}+\pi N\left(r_{0}^{4}-r_{i}^{4}\right) \frac{\mu_{0}}{d} \dot{\theta}
$$

Furthermore, in order to produce the electric field, a positive voltage is applied to the fixed plates, while the rotating plates, which geometrically alternate with the fixed ones, are grounded. The field is then the same in all the inter-plate gaps, and is simply given by:

$$
E=\frac{V}{d}
$$

The final model can then be written as:

$$
T=\left(\alpha_{0}-\alpha_{1} \dot{\theta}\right) V^{2}+\alpha_{2} \dot{\theta}
$$

where $\alpha_{i}, i \in[1, \ldots 3]$ are constant positive scalars given by:

$$
\left\{\begin{array}{l}
\alpha_{1}=\frac{\pi 4 \mathrm{NC}_{\mathrm{d}}\left(\mathrm{r}_{0}^{3}-\mathrm{r}_{\mathrm{i}}^{3}\right)}{3 \mathrm{~d}^{2}} \\
\alpha_{1}=\frac{\pi \mathrm{NC} \mathrm{C}_{\mathrm{v}}\left(\mathrm{r}_{0}^{4}-\mathrm{r}_{\mathrm{j}}^{4}\right)}{\mathrm{d}^{3}} \\
\alpha_{2}=\pi \mathrm{N}\left(\mathrm{r}_{0}^{4}-\mathrm{r}_{\mathrm{i}}^{4}\right) \frac{\mu_{0}}{\mathrm{~d}}
\end{array}\right.
$$

\section{EXPERIMENTAL PARAMETER IDENTIFICATION}

Our objective here is to verify the ability of Equation (13) to describe the post-yield behaviour of the actuator and experimentally identify the model parameters. First experiments are done with a constant speed of the DC motor that provides the input torque to the FP actuator. Then the same experiments are performed with different constant speeds. In order to avoid the excitation of internal hysteretic cycles, that would corrupt the parametric identification; a careful repetitive procedure is used as follows for each experiment with constant speed:

a) the actuator is initially placed at $\theta=0^{\circ}$;

b) the motor starts and obtains a constant velocity, and a ramp voltage is applied to the ERF. It is verified that the ramp slope is slow enough compared to the expected actuator dominant dynamics. As a result, a quasi-static behaviour can be assumed. When applying the ramp voltage, the resistive torque is measured;

c) when this experiment is over, the actuator is put back in the initial position with a null electric field;

d) it is then verified that, redoing the experiment from step a, the torque response is repeatable.

On Figure 4, lines in blue show typical results of the torque-voltage relationship for two different velocities of the DC motor.
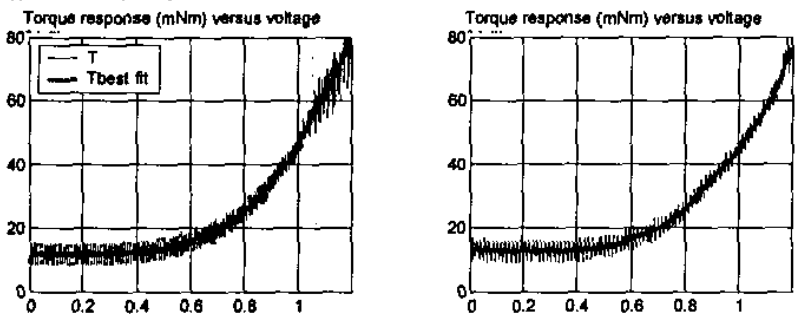

Fig. 4: Reactive Torque Response vs. Velocity.

From Figure 4 it can be verified that the response shape agrees with Equation (13). It can be noticed that, below a certain voltage limit $V_{\text {lim }}$, which depends on the velocity, the ERF seems reactionless. Note that this is in accordance with Equation (4), which describes the static pre-yield behaviour, although what is measured here is the dynamic mode. To account for this experimentally emphasized phenomenon, and taking into account that the experiments were performed with constant speed, then Equation (13) could be written as:

$$
\mathrm{T}=\left\{\begin{array}{llr}
\mathrm{T}_{0} & \text { if } \quad \mathrm{V}<\mathrm{V}_{\text {lim }} \\
\mathrm{T}_{0}+\mathrm{k} & \left(\mathrm{V}-\mathrm{V}_{\text {tim }}\right)^{2} \quad \text { otherwise }
\end{array}\right.
$$

where $T_{0}$ is the minimal torque measured with a zero voltage, $V_{\text {lim }}$ is the voltage under which nothing happens.

The three parameters of a constant speed experiment, namely $T_{0}, k$, and $V_{\text {lim }}$ are then computed in order to obtain a least square best fit. In Figure 4, the red lines show the best fits of this model for the different velocities. The identified parameters $T_{0}, \mathrm{k}$, and $V_{\text {lim }}$ are different for each experiment, which denotes their dependency on velocity. In turn, a best fit is used to express the velocity dependency of these three parameters. It is found that the three parameters are linear functions of the velocity. Namely:

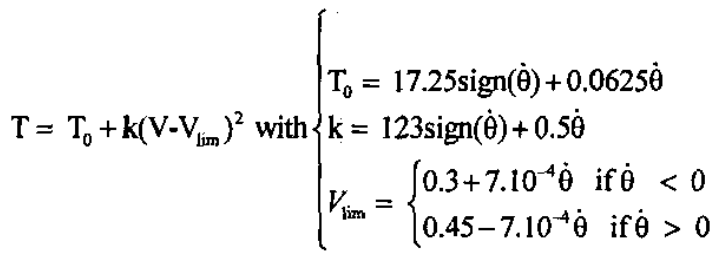

where $\mathrm{V}$ is the computer output voltage expressed in Volts (which is also the ERF applied voltage expressed in $\mathrm{kV}$ ) and the torques are expressed in $\mathrm{mNm}$. Note that the 
velocity dependency confirms, in part, the theoretical model. For example, the coefficient $k$ decreases with the velocity, as the expected effect of the positive scalar $\alpha_{1}$. Some discrepancies can also be noticed. For example, the zero field torque $T_{0}$ is not reduced to a pure viscosity, as expected. Rather, there is a dry friction term $(17.25 \mathrm{mNm})$.

Figure 5 shows the 3D plot mapping of the FP actuator resistive torque output as a function of the applied voltage (V) and of the DC motor velocity $\dot{\theta}$ using the experimental identified model. Experimental results have been superimposed with different colours for different velocities. Experimental curves fit well the model's 3D plot mapping, which illustrate the accuracy of the identified model. Again, recall that this accuracy is obtained thanks to a careful procedure that was aimed at preventing hysteretic internal cycles from occurring. Should hysteretic cycle occur, the model would not be as precise as depicted by Figure 5 .

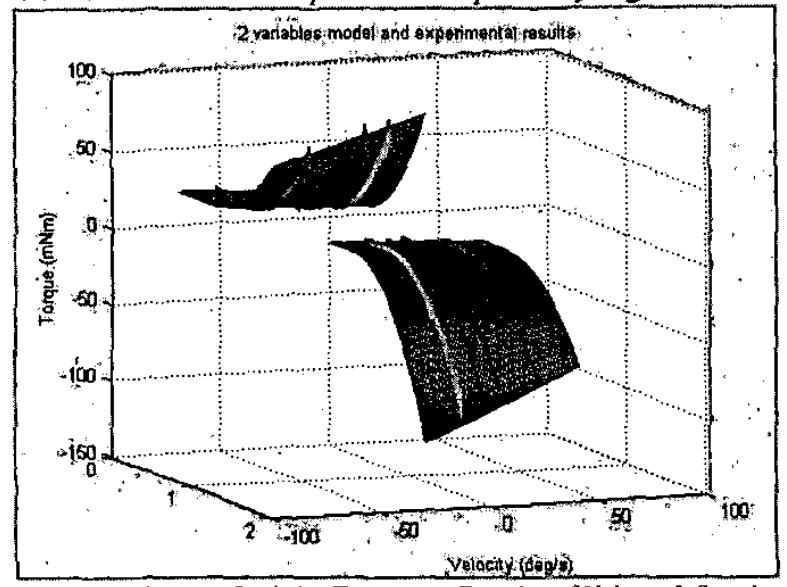

Fig. 5: FP Actuator Resistive Torque as a Function of Voltage \& Speed.

\section{OPEN LOOP CONTROL}

To evaluate the possibility of using an ERF haptic device without any torque sensor measurements, open loop control experiments were performed and are reported in this section. Avoiding torque measurement will considerably simplify the design and control and reduce the cost of the device. The open loop controller will be using the actuator model identified with Equation (16) to convert desired torques to input voltages in the actuator. To do so Equation (16) needs to be "inversed" so that it calculates the voltage $\mathrm{V}$ as a function of the command torque $\overline{\mathrm{T}}$ and of the actuator velocity $\dot{\theta}$ :

$$
\mathrm{V}(\tilde{\mathrm{T}}, \dot{\theta})=\mathrm{V}_{\mathrm{lim}}(\dot{\theta})+\sqrt{\frac{\tilde{\mathrm{T}}-\mathrm{T}_{0}(\dot{\theta})}{\mathrm{k}(\dot{\theta})}}
$$

To be able to use Equation (17) the following constraint needs to be satisfied:

$$
\mathrm{T}(\mathrm{V}, \dot{\theta})-\mathrm{T}_{0}(\dot{\theta}) \geq 0
$$

Inequality (18) means that the desired resistive torque that the actuator should produce must be larger than $T_{0}(\dot{\theta})$, i.e. the minimal torque measured with a zero voltage, which is the sum of a dry friction term and a viscous term depending on the velocity. In cases where the desired torque is such that Inequality (18) is not satisfied, i.e. when the applied voltages are between zero and $V_{\text {lim }}$, a linear interpolation is used to calculate the applied voltage:

$$
\mathrm{V}(\tilde{\mathrm{T}}, \dot{\theta})=\mathrm{V}_{\text {lim }}(\dot{\theta}) \frac{\tilde{\mathrm{T}}}{\mathrm{T}_{0}(\dot{\theta})}
$$

Equation (19) does not correspond to any physical model, but it is used in the open and closed loop control to provide model continuity when the voltages are lower than the voltage $V_{\text {lim }}$ where the ERF is reactionless.

In Equation (17) we need to note that the sign of the desired torque has no importance for control purposes even though the physical values of the torques can be positive or negative based on the direction of the motor rotation. Since the actuator is semi-active and can only resist external torques by increasing its viscosity, for the same voltage input, it will resist with a positive or negative torque depending on how it is excited by the operator. For this reason, when developing the torque controller, the absolute value of the desired or measured torque will be used. Thus, Equation (17) is used with positive (i.e. absolute) values for the desired torque.

A series of open-loop control experiments were performed where a ramp function was used as the desired torque while the motor run at several constant velocities (see Figure 6).
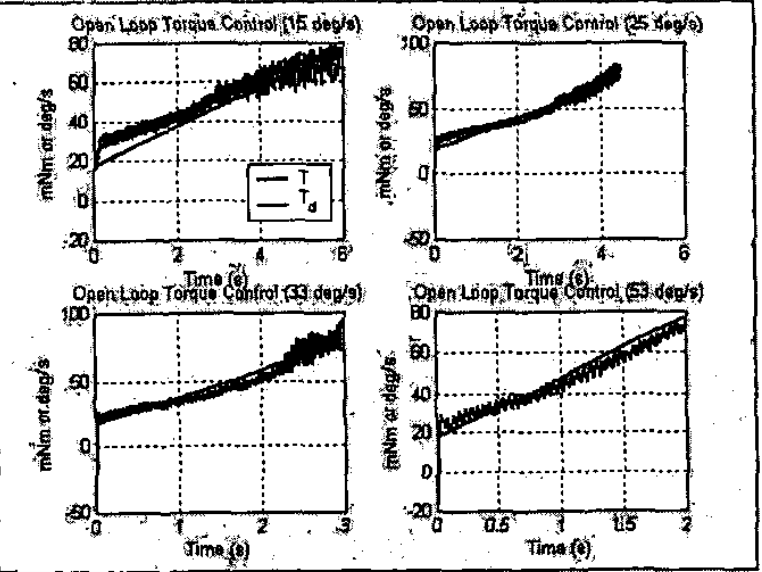

Fig. 6: Open Loop torque Control With Positive Torques.

The output voltage was computed using Equation (17). The resistive torque of the FP actuator was measured using the torque sensor and compared to the desired one. Even though the output resistive torque exhibits a reasonable accuracy which can be adequate for several applications, in certain instances the error between the desired and measured torques can reach up to $20 \mathrm{mNm}$ (such as for 
desired torques of $80 \mathrm{mNm}$ ). For a fine haptic application, this error is too large. In addition, should the experimental conditions vary (e.g. due to hysteresis or temperature changes) the error between the desired and actual torque outputs would increase. Therefore, the conclusion from the open-loop control experiments is that a closed-loop control would be necessary to finely control the resistive torque of the FP actuator.

\section{CLOSED LOOP CONTROL}

Figure 7 shows the block diagram of a non-linear, model based, PI controller with a feed-forward term. A control error is fed into a PI controller. The command torque is the sum of the output from the PI controller and a desired torque feedforward term. The latter is used so that the PI compensator works with small values of inputs and outputs. The command torque is converted to a control voltage using the non-linear Equation (17). Some representative results from the experiments with this controller are shown in Figure 8. It can be clearly seen that the response is fast (settling time is approx. $70 \mathrm{~ms}$ ) and well damped. The response is reproducible for any value of the desired torque input, which indicates a successful linearization. Extensive experiments have demonstrated a strong robustness of the controller to variations of experimental conditions, such as the magnitude and sign of velocity. The closed loop dynamics are rather constant, even when the actuator is cycled arbitrarily, i.e. when its hysteretic behaviour is excited.

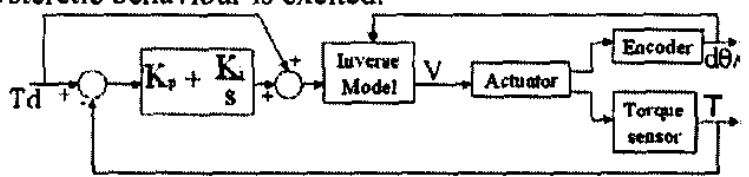

Fig. 7: Non Linear PI Controller With Feed-Forward Term.
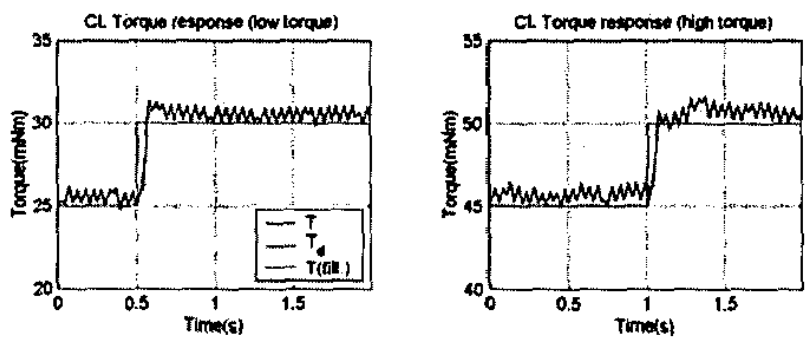

Fig. 8: Closed loop Step Response Using a Non-Linear PI Controller.

\section{CONCLUSIONS}

This paper studied the closed-loop torque control of ERF based actuators. To do this, an experimental method has been proposed to identify the behaviour of the system. This model describes the resistive torque as a function of both the applied voltage and the velocity, and confirms the general trends of the theoretical model. Next, torque control of an ERF based actuator was investigated. The experimental inverse model was added to a PI controller in order to linearize the system. It has been shown that this non-linear PI controller with feed-forward exhibits a precise and accurate response, making it well-suited for haptic applications. In our current work we are investigating the possibility of using semi-active, ERF based actuators in haptic devices.

\section{ACKNOWLEDGMENT}

This work was supported by the National Science Foundation (DMI-9984051, CMS-0301338, CMS0422720). Any opinions, findings, conclusions or recommendations expressed in this publication are those of the authors and do not necessarily reflect the views of the National Science Foundation.

\section{REFERENCES}

1. Phule P. and Ginder J., "The Materials Science of Field-Responsive Fluids," MRS Bulletin, Aug. 1998, pp. 19-21.

2. Mavroidis C., Bar-Cohen Y. and Bouzit M., "Chapter 19: Haptic Interfaces Using Electrorheological Fluids", Invited Chapter in Electroactive Polymer (EAP) Actuators as Artificial Muscles: Reality, Potentials and Challenges, Y. Bar-Cohen Editor, SPIE Optical Engineering Press, February 2001, pp. 567-594.

3. Choi SB., "Control of ER devices", International Journal of modern physics $B$ vol 13 Nos 14,15 and 16, (1999) 2160-2167.

4. Powell JA., "ERF as a Means of Vibration Suppression", Proc. of Int. Conf. on Vibration and Noise, April 25.27, 1995, pp 1-8.

5. Rettig U. and Von Stryk O., "Numerical Optimal Control Strategies for Semi-Active Vehicle Suspension with ERF Dampers" In: K.-H. Hoffmann, R.H.W. Hoppe, V. Schulz (eds.): Fast Solution of Discretized Optimization Problems (Birkhauser Verlag 2001) pp. 221-241.

6. Gavin HP., "Control of Seismically-Excited Vibration Using ER Materials and Lypunov Methods" IEEE Transactions and Automatics Control, Vol. 9, No. 1, pp. 27-36, 2001.

7. Nakano M., Minagawa S., and Hagino K., "PMW Flow Rate Control of ER Valve and its Application to ER Actuator Control" International Journal of Modern Physics B Vol 13 Nos 14-16, (1999), pp. 2168-2175.

8. Mavroidis C., Pfeiffer C., Celestino J. and Bar-Cohen Y., "Design and Modeling of an Electro-Rheological Fluid Based Haptic Interface," Proceedings of the 2000 ASME Mechanisms and Robotics Conference, Baltimore, MD, September 10-13, 2000. Paper DETC2000/MECH-14121.

9. Melli-Huber J., Weinberg B., Fisch A., Nikitczuk J., Mavroidis C., Wampler C," "Electro-Rheological Fluidic Actuators for Haptic Vehicular Instrument Controls", Proc. of the Eleventh Symposium on Haptic Interfaces for Virtual Environment and Teleoperator Systems, March 22 and 23, 2003, Los Angeles, CA, pp. 262-267.

10. Block, H. and Kelly, J. P., "Electro-Rheology", Journal of Physics, D: Applied Physics, Vol. 21, 1988, pp. 1661

11. Rajagopal KR., Ruzicka M., "Mathematical Modelling of ER Materials", Continuum Mech. Thermodyn. 13: pp. 59-78.

12. Bonnecaze and Brady, "Yield Stresses in Electrorheological Fluids", Journal of Rheology, 36,73-115, 1992.

13. Wang and Gordaninejad, "Flow Analysis of Field-Controllable, Electrorheological and Magnetorheological Fluids Using Hersche]Bulkley Model", J. of Intelligent Materials, Systems and Structures, Vol.10, pp. 601-608, 1999.

14. Smart Technology Ltd, "Technical Information Sheet - ElectroRheological Fluid LID 3354S", 2001. 